Voix et Images

voixetimages

\title{
Petits livres sur de vastes sujets
}

\section{Robert Major}

Volume 19, numéro 3 (57), printemps 1994

Science et fiction au Québec : L’émergence d'un savoir

URI : https://id.erudit.org/iderudit/201122ar

DOI : https://doi.org/10.7202/201122ar

Aller au sommaire du numéro

\section{Éditeur(s)}

Université du Québec à Montréal

ISSN

0318-9201 (imprimé)

1705-933X (numérique)

Découvrir la revue

Citer cet article

Major, R. (1994). Petits livres sur de vastes sujets. Voix et Images, 19(3), 628-635. https://doi.org/10.7202/201122ar d'utilisation que vous pouvez consulter en ligne.

https://apropos.erudit.org/fr/usagers/politique-dutilisation/ 


\section{Essai}

\section{Petits livres sur de vastes sujets}

\section{Robert Major, Université d'Ottawa}

Autrefois, il me semble, lorsque les communautés religieuses contrôlaient à la fois l'enseignement et les maisons d'édition, il y avait pléthore d'éditions littéraires visant un public scolaire. Anthologies, manuels et guides divers foisonnaient, ainsi, d'ailleurs, que les éditions bon marché de classiques québécois. Nous avons tous, sur nos rayons de bibliothèque, des témoins jaunissants et friables de cet effort constant, massif, d'éducation littéraire.

Qui n'était pas sans ambiguïté, j'en conviens. Combien d'anthologies et de textes, scrupuleusement expurgés, avaient un objectif moral et patriotique davantage que littéraire; et combien d'œuvres étaient mises à l'écart par une pensée religieuse maladivement rigoriste ou pusillanime. Mais, sans doute, l'expurgation et la censure étaient-elles un moindre mal: À tout prendre, mieux vaut, pour un adolescent boutonneux, pouvoir se procurer une édition imparfaite de Maria Chapdelaine ou une anthologie de textes tronqués, pouvoir lire une version adoucie d'Arthur Buies ou de Louis Fréchette, que de pas avoir du tout d'édition à portée de sa bourse. Ces nourritures édulcorées étaient nourritures néanmoins, et pouvaient conduire loin, du moment qu'on y avait goûté.

Il est de bon ton, depuis plusieurs années, de se moquer de ces efforts lénifiants ou de crier haro sur toute entreprise qui pourrait évoquer un avatar lagardémichardesque. Les nouveaux censeurs, ceux de la pureté et de l'intégrité littéraires - car pour ce qui est de l'intégrité de la jeune personne, on n'en a cure -, oublient trop facilement ce qu'ils doivent eux-mêmes à cette formation: lacunaire, certes; imparfaite, c'est évident; et bête, même, par moments, mais qui créait, néanmoins, les conditions de son dépassement. Qui peut prévoir les effets du cri de Menaud sur un lecteur impressionnable?. On l'a vu, d'ailleurs, en 1980, quand Menaud n'a pas reconnu ses propres enfants. Et si l'on donnait à lire, jadis, une page d'Alfred Desrochers, sous prétexte de son régionalisme réactionnaire, peut-être aussi ouvrait-on grande la porte à ses hommes du Nord, ivrognes, sacrards, 
démesurés, race surhumaine dont on ne pouvait guère, par la suite, contenir l'influence. Ce que feront de leurs lectures les écoliers, qui peut le prévoir? Encore faut-il leur donner à lire.

Il me semble que l'institution littéraire québécoise, telle qu'elle s'est constituée depuis une trentaine d'années, sous l'impulsion des universitaires et sous la gouverne des instances subventionnaires, et telle qu'elle s'active présentement sous les diktats de la Recherche, a scandaleusement négligé un grand pan indispensable à toute littérature nationale: la préparation, pour de jeunes lecteurs, d'une part des textes dans des éditions adaptées, et d'autre part des outils indispensables à la compréhension de ceux-ci. Où sont les éditions commentées des classiques québécois? les monographies d'écrivains? les biographies littéraires? les manuels bien faits et abordables? les anthologies franchement scolaires? À des prix d'étudiants, évidemment. En somme, les ouvrages dont toute littérature ressent le besoin aussitôt qu'elle a compris que son destin est en partie lié à la pédagogie et aux programmes scolaires? Tout cela tarde à venir. Parce que cela n'est guère subventionnable?

C'est pourquoi il faut accueillir avec enthousiasme des entreprises comme la collection Boréal Express, qui semble s'être donné pour mandat de fournir de petits guides intéressants et peu coûteux sur une diversité de questions, quelques-unes relatives à la littérature. Parmi les titres tout récents ${ }^{1}$, j'en retiens un qui pourrait être d'intérêt particulier pour les littéraires: Fernande Roy, Histoire des idéologies au Québec aux $X X^{e}$ et $X X^{e}$ siècles ${ }^{2}$, et je profite de l'occasion pour rappeler un ouvrage de cette collection publié depuis plus de deux ans déjà, mais qui n'a pas été l'objet d'une recension dans ces pages: Le Roman québécois de Réjean Beaudoin ${ }^{3}$.

Le livre de Fernande Roy est l'ouvrage d'une historienne et non d'une sociologue, d'une politicologue ou d'une littéraire. Cela n'est pas sans conséquences. En effet, on la sent plus à l'aise dans l'histoire politique et plus attirée par les hommes politiques que par les groupes sociaux, les expressions socioculturelles de ces groupes ou encore les enjeux économiques. L'importance accordée à un politique comme Laurier, par exemple, est assez révélatrice à cet effet. Or, on sait que les partis politiques sont agités par les grands débats idéologiques et, à la limite, qu'ils incarnent, très grossièrement, un certain nombre d'options idéologiques. Mais dans le système essentiellement bi-partite 
ou tri-partite qui est le nôtre depuis le $\mathrm{xIx}^{\mathrm{e}}$ siècle, les partis sont davantage de vastes coalitions englobantes, mouvantes, difficilement saisissables, que l'expression d'une idéologie précise; davantage préoccupés par la boîte de scrutin (aujourd'hui: par le dernier sondage) que par la cohérence idéologique. Les partis, dans leur pragmatisme calculateur, ont l'idéologie (ou plutôt la mixture idéologique) qui leur vaudra le succès électoral. C'est ainsi une des limites sérieuses de ce petit livre que de souvent définir le débat idéologique par la polémique politique et électorale qui n'est qu'un effet, souvent fort distancié, des options idéologiques fondamentales. Sans doute est-ce en partie aussi pourquoi ce livre accorde peu d'importance à des courants idéologiques récents, d'importance collective évidente (pacifisme, écologisme, contre-culture, individualisme narcissique, etc.), mais dont l'effet électoral n'est pas facile à voir.

Cela dit, toutefois, l'ouvrage sera utile pour son survol et sa synthèse. Après une brève introduction qui définit l'idéologie (vraisemblablement dans la foulée de Jean Baechler) comme un ensemble coordonné de valeurs, d'idées, de symboles, forcément polémique, qui mobilise en vue de l'action et vise essentiellement le pouvoir, l'ouvrage présente l'évolution des idéologies québécoises depuis la fin du régime français jusqu'à l'époque contemporaine. Les limites du volume et le public visé imposent un certain schématisme et de nombreux raccourcis, et interdisent un certain nombre de choses. On ne trouvera pas l'effet percutant des analyses de Marcel Rioux, ni la profondeur de celles de Fernand Dumont, ni l'ampleur de celles de Denis Monière. On trouvera, toutefois, une assez bonne exploitation de chacune ${ }^{4}$, et quelques aspects particuliers, intéressants à signaler. Une certaine distance critique, par exemple. Voici une historienne des idéologies qui est pourtant prête à admettre que "ce ne sont pas seulement les idées ou les idéologies qui mènent le monde" (p. 33). Cet esprit critique la portera, ailleurs, à juger sévèrement Cité libre. Non pour ses options idéologiques, mais surtout pour ses prétentions d'originalité et pour sa myopie historique (p. 101).

Les reproches adressés à Cité libre jettent un certain éclairage sur le fil conducteur de ce petit livre, nettement exposé, d'ailleurs, dans une note qui précède la bibliographie:

La majorité des ouvrages qui suivent ont été écrits alors que la croyance au monolithisme idéologique de la société québécoise - et donc l'absence de libéralisme - entre 1867 et 1945, voire 1960, était largement répandue. Comme aux auteurs cités dans les notes, je leur ai beaucoup emprunté, tout en proposant souvent une lecture différente (p. 125). 
La "lecture différente" découle de la conviction que le libéralisme est une idéologie constamment présente, depuis la fin du XviII ${ }^{\mathrm{e}}$ siècle jusqu'à nos jours, mais sous de multiples avatars. Ses valeurs (liberté, sécurité, propriété privée, individualisme) gouvernent même l'action d'un Duplessis, par exemple. Il n'y a donc pas eu de monolithisme idéologique au Québec, contrairement aux prétentions de nombreux historiens (et aux rabâchages d'innombrables critiques littéraires!). La lecture de la presse à grand tirage indique très clairement que même pendant les périodes qui sont censées être celles du conservatisme débridé et du messianisme homogène, les valeurs libérales occupent le devant de la scène ou traversent en filigrane les différents discours.

À ce libéralisme polymorphe se combinait un nationalisme tout aussi protéiforme, ce dernier, d'ailleurs, épousant diverses idéologies, se prêtant à d'étranges alliances au cours des siècles et, de façon générale, compliquant constamment le tableau. Par ailleurs, face au libéralisme changeant, se dresse depuis toujours l'Église, essentiellement conservatrice et communautaire. Ces deux forces antagoniques structurent le survol historique qui est présenté par Fernande Roy.

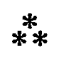

Réjean Beaudoin, dans son petit livre sur Le Roman québécois, n'a pas l'ambition d'effectuer une présentation historique, ni de "faire le tour du roman québécois" (p. 12). Entreprise impossible dans les limites qui sont les siennes. Le roman est le genre le plus répandu, au Québec comme ailleurs en Occident. Il est varié, disparate, multiforme, l'objet de pratiques très diverses. "Comment ordonner un tel foisonnement?" (p. 11), et d'autant plus que la production ne cesse de croître. S'il s'est publié deux cents romans entre 1837 et 1915, le Québec en publie maintenant autant chaque année!

Reconnaissons donc à l'auteur le mérite et le courage de cette entreprise de débroussaillage et de classement, et d'autant plus que pour chacun de ses lecteurs (j'en suis, comme on le verra) il aura forcément tort, puisqu'il aura oublié ceci ou simplifié cela. De plus, ce travail est effectué avec une certaine originalité, avec un point de vue résolument contemporain, car l'auteur ne s'intéresse qu'aux romans vivants, c'est-à-dire lus, "lisibles" ou influents aujourd'hui. "Ainsi, notre point de départ n'est autre que la situation actuelle, c'est-à-dire l'ensemble des œuvres, anciennes et récentes, qui forment aujourd'bui cet espace de lecture et d'écriture particulier qu'on appelle le roman québécois" (p. 12-13). 
L'ouvrage est divisé en sept chapitres. Le premier trace "l'évolution du genre", mais en reculant dans le temps: à partir de la période contemporaine, l'auteur mentionne les romans que le présent a récupérés. Le deuxième chapitre oppose "romans du groupe et romans de l'individu ", c'est-à-dire les romans centrés sur les valeurs collectives et les romans qui font la critique de ces valeurs. Le troisième confronte "romans du territoire et romans de l'espace", c'est-à-dire les romans de l'enracinement et de la possession, par opposition aux romans de l'espace et de l'aventure, et cherche ainsi à "dessiner la géographie imaginaire du roman québécois " (p. 45). Le quatrième chapitre aborde, sous le titre "les mots et les choses", la tension "entre la volonté de nommer une réalité spécifique et le besoin d'assumer une nouvelle liberté d'écrire" (p. 59), tandis que le cinquième, sous le titre "le même et l'autre", étudie la question de l'altérité en examinant les figures de l'Amérindien et de la femme et la représentation de différentes communautés culturelles. Les deux derniers chapitres, quant à eux, sont en quelque sorte périphériques. "Le roman québécois et les médias" (chapitre six) fait état des grands succès de librairie et des adaptations (pour la radio, la télévision, le cinéma), pour finalement déplorer que le roman soit de plus en plus médiatisé, gagné par le monde du spectacle et réduit à un objet de consommation. Par ailleurs, "le roman québécois et la critique" (chapitre sept) signale les principales approches méthodologiques du genre (historique, sociologique, herméneutique) et leurs praticiens. L'épilogue, finalement, offre au lecteur un peu de prospective et lance un cri d'alarme devant les pressions exercées sur le roman par la culture de masse et le monde du spectacle et devant l'incapacité de l'école de former adéquatement de nouveaux lecteurs, aptes à aborder un genre souvent difficile et complexe.

Cette composition particulière permet à l'auteur d'effectuer assez librement des rapprochements ou des regroupements et d'y aller avec beaucoup de souplesse, coiffant souvent ses recoupements ou ses brèves analyses de titres ou sous-titres éloquents, riches de significations, quelquefois accrocheurs et provocants, au risque d'être injustes et partiaux. Dans l'ensemble le canevas reçoit notre assentiment, du moment qu'on accepte qu'un petit livre de ces dimensions ne saurait tout dire et sera forcément conduit à effectuer des choix.

Par ailleurs, on acquiesce beaucoup plus difficilement à une simplification regrettable comme celle présentée dans le premier chapitre de survol, lorsque la période de 1837 à 1916 est coiffée du titre: "Le degré zéro de l'écriture". Pour l'auteur, six œuvres (à peine) de cette "ingrate et longue période de gestation" "surnagent et gardent une certaine actualité . Pour le reste, 
il s'est écrit des romans de colonisation, des romans historiques, des épopées sans héros, des romans d'exil intérieur, des aventures très diverses auxquelles manquera toujours la qualité du style. C'est une écriture pauvre, censurée, placée sous la haute surveillance d'une instance idéologique qui veille à l'orthodoxie du patriotisme comme au plus précieux des biens, quand il ne reste justement aucun autre bien. C'est vraiment le degré zéro du roman (p. 25).

On peut s'étonner d'une telle évaluation. Elle reprend les sempiternels propos sur le monolithisme idéologique de la période (la lecture de Fernande Roy s'avère de plus en plus urgente...), indique une lecture superficielle des œuvres et une méconnaissance du fonctionnement du texte littéraire (qui le plus souvent laisse des interstices dans sa masse afin de subvertir ce qui est ostensiblement l'objet de son propos), postule vraisemblablement la "qualité du style" des œuvres de notre époque (alors que les manifestations contemporaines d'une écriture pauvre, sous haute surveillance des recettes, me semblent assez évidentes). Ce jugement, en somme, rend le plus mauvais service aux éventuels lecteurs qui souhaiteraient un guide plus sensible aux caractéristiques propres d'une tradition littéraire, à redécouvrir ou à réinventer par chaque génération.

Sans doute faudrait-il parler du degré zéro de la critique. Un tel jugement me rappelle celui de certains partipristes qui, dans la ferveur de l'éclosion d'une modernité québécoise, jugeaient sévèrement les œuvres du $\mathrm{XIX}^{\mathrm{e}}$ siècle. Laurent Girouard, par exemple, qui disait des Anciens Canadiens que c'était le "roman d'un sénile", mal écrit, "mièvre, d'une connerie à toute épreuve" (Parti pris, vol. I, n ${ }^{\circ} 1$, mars 1964). André Brochu, qui avait plus de sens critique et sans doute beaucoup plus d'érudition, s'empressait, toutefois, dès l'année suivante, de réhabiliter les œuvres qui composent notre tradition, et entre autres Les Anciens Canadiens, affirmant qu'il y avait des choses à reconnaître dans une telle œuvre, par exemple la "lyrique souterraine extrêmement riche, centrée sur le thème du fleuve. (Parti pris, vol. II, no 5 , janvier 1965).

C'était il y a trente ans! Le jugement à l'emporte-pièce d'un critique amateur comme Girouard était compréhensible dans les circonstances. Mais aujourd'hui? Le degré zéro de la critique, c'est répéter des jugements rapides et faciles, sans se donner le mal d'une lecture qui dépasserait les premières évidences. Il n'y a que le Québec pour condamner, au silence ou au mépris, les œuvres de son passé et pour détourner les jeunes de leur lecture. Comme si on se haussait en rabaissant ses pères. Les œuvres de notre tradition sont à relire ${ }^{5}$ et non pas à rejeter ainsi en bloc. Où se situe vraiment le monolithisme: au $\mathrm{XIX}^{\mathrm{e}}$ ou dans sa réception actuelle par la critique? 
D'ailleurs, si l'auteur était simplement fidèle à sa propre perspective de lecture (qu'est-ce qui se lit dans le présent? qu'est-ce qu'on trouve en librairie et pas seulement en bibliothèque? qu'est-ce qui est texte représentatif?), force lui serait d'admettre que de nombreux romans du XIX ${ }^{\mathrm{e}}$ siècle sont plus lus que ceux de notre époque. En effet, ce n'est pas faire injure à Yvon Rivard, à Pierre Nepveu ou à Nicole Brossard que de reconnaître que leurs romans ont probablement moins de lecteurs aujourd'hui que plusieurs œuvres du XIX ${ }^{\mathrm{e}}$ siècle n'en ont. De plus, si "l'influence repérable" (p. 12) d'un roman est un critère important pour le retenir dans la "description raisonnée " qu'est cet ouvrage, on peut s'étonner qu'un livre comme Jean Rivard, à l'origine de tout un mouvement littéraire et qui, de plus, renaît sous la plume de Réjean Ducharme, ne soit pas mentionné et retenu. Quand un écrivain contemporain majeur fait son "espace de lecture et d'écriture" d'un roman du $\mathrm{XIX}^{e}$ siècle, peut-être cela devrait-il mettre la puce à l'oreille des critiques. Mais il faut dire que, sur ce plan, les écrivains québécois ont plus d'ouverture et de perspicacité que leurs commentateurs. Jacques Ferron, dans sa vaste et libre érudition qui ne négligeait aucune œuvre, même les plus humbles, est certainement l'illustration exemplaire de cette intelligente prospection de notre tradition. Plaise au ciel que son exemple soit davantage suivi par la critique!

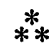

Le livre de Réjean Beaudoin invite à un rapprochement avec un petit livre publié en anglais au même moment, et portant sur le même sujet: celui de Ben-Z. Shek, French Canadian \& Québécois Novels ${ }^{6}$. Rapprochement d'autant plus pertinent que les Québécois auraient peut-être intérêt à considérer ce que les Canadiens réalisent dans le domaine des livres utiles. Les livres ou les collections pratiques sur la littérature canadienne sont nombreux et de qualité. Citons, à titre d'exemple, le Oxford Companion to Canadian Literature (en un volume, ce qui en fait un compagnon réel, un vade mecum authentique); les collections "Reappraisals: Canadian Writers", "Canadian Writers and their Works" de ECW, la "Canadian Collection" au sein de la "Twayne Series", "Studies in Canadian Literature", "Critical Views on Canadian Writers" et l'ancienne collection de McLellan and Stewart: "The New Canadian Library Writers Series". Autant de petits ouvrages qui, parmi d'autres, rendent de précieux services aux étudiants de "Canlit".

Mais la critique anglophone soucieuse de pédagogie ne néglige pas pour autant la littérature québécoise. Témoin ce livre de Ben-Z. 
Shek, remarquablement bien fait et qui n'a malheureusement pas son pendant en français. L'étude est, d'une part, un survol du roman québécois, en cinq chapitres bien tassés; d'autre part, l'auteur présente, à l'intérieur de cet ensemble de considérations historiques et critiques, de nombreuses analyses, succinctes mais denses, fermes mais nuancées, d'œuvres représentatives.

Les chapitres couvrent la période de gestation (1837-1937), l'éclosion du roman moderne (1938-1959), les années soixante, l'émergence du "je" féministe, et finalement les vingt dernières années. Les ouvres représentatives analysées à l'intérieur de ces périodes sont bien choisies, et offrent un échantillonnage fort diversifié. L'auteur présente un résumé critique du roman, met celui-ci en relation avec la société du temps et son contexte idéologique, présente un certain nombre de renseignements biographiques sur l'auteur ou historiques sur le texte et sa composition, établit des relations avec des œuvres contemporaines ou comparables. Cet ensemble de précisions critiques et érudites éclairent le roman choisi comme on le souhaite d'un guide de lecture. Aucune surprise dans ces analyses, mais aucune facilité non plus, et jamais de tape-à-l'œil. Simplement beaucoup de métier et un sens critique éprouvé ${ }^{7}$.

À quand une traduction en français, pour nos étudiants et pour nous?

1. Dont: Edith Madore, La Littérature pour la jeunesse au Québec, Montréal, Boréal, coll. "Boréal Express", nº 9, 1994, 127 p.; Mira Falardeau, La Bande dessinée au Québec, Montréal, Boréal, coll. "Boréal Express", nº6, 1994, 126 p.

2. Fernande Roy, Histoire des idéologies au Québec aux $x x^{e}$ et $x x^{e}$ siècles, Montréal, Boréal, coll. «Boréal Express", n 8, 1993, 127 p.

3. Réjean Beaudoin, Le Roman québécois, Montréal, Boréal, coll. "Boréal Express", $\mathrm{n}^{\mathrm{o}} 3,1991,126 \mathrm{p}$.

4. Curieusement, ni Rioux, ni Monière ne figurent dans la bibliographie. Ni, d'ailleurs, Royer, Hare, Ryerson, Hamelin et Roby, etc.

5. D'ailleurs, pour l'essentiel, Réjean Beaudoin reprend les idées de son livre de 1989 , livre qui avait tendance à simplifier le xix ${ }^{\mathrm{e}}$ siècle ou du moins à ne pas percevoir la complexité des débats idéologiques qui l'agitaient. J'avais eu l'occasion d'exprimer des réserves sur ce livre également (Voix et Images, $\mathrm{n}^{\circ} 46$ ).

6. Ben-Z. Shek, French-Canadian \& Québécois Novels, Toronto, Oxford University Press, coll. "Perspectives on Canadian Culture", 1991, 151 p.

7. Un reproche, toutefois. La tendance à voir des calembours partout dans les noms des personnages de notre univers romanesque. 